


\title{
DEVELOPMENT OF THE PROFILE OF EQUILIBRIUM OF THE SUBAQUEOUS SHORE TERRACE
}

A Dissertation SUbmitted to the faculties of the graduate SCHOOLS OF ARTS, LITERATURE, AND SCIENCE, IN CANDIDACY FOR THE DEGREE OF DOCTOR OF PHILOSOPHY

$$
\text { (DEPARTMENT OF GEOLOGY) }
$$

N. M. FENNEMAN

\author{
PRINTED BY
}

\section{Tbe Univergitp of Cbicago Dress}

\section{CHICAGO}


GD451

F4

Digitized by the Internet Archive in 2008 with funding from Microsoft Corporation 


\section{DEVELOPMENT OF THE PROFILE OF EQUILIBRIUM OF THE SUBAQUEOUS SHORE TERRACE.}

THE profile of a shore as seen at any one time is a compromise between two forms. One of these is the form which it possessed when the water assumed its present level; from this form it is continually departing. The other is the form which the water is striving to give to it ; toward this form it is continually tending. There is a profile of equilibrium which the water would ultimately impart, if allowed to carry its work to completion. The continual change of shore line and the supply of new drift are everchanging conditions with which no fixed form can be in equilibrium. There are, however, certain adjustments of current, slope and load which, when once attained, are maintained with some constancy. The form involved in these adjustments is commonly known as the profile of equilibrium. When this profile has once been assumed the entire form may slowly shift its position toward or from the land, but its slope will change little or not at all. It may be compared to a stream channel which has reached grade but not base level.

The force which the water exerts is derived ultimately from the wind. The immediate agencies in the work are waves and currents. It will be convenient to consider these first as acting independently of the wind which caused them, and second, as acting under its continuous influence. It is also desirable to 
consider waves first in their free forms, while meeting no resistance and hence doing no external work. This condition is found in deep water. The various ways in which the bottom or shore may offer resistance and be subject to work may then be discussed.

\section{WAVES IN WATER OF INFINITE DEPTH .}

When wave agitation does not reach the bottom of a body of water it is customary to speak of the depth as infinite, because the wave is not influenced by the existence of a bottom.

PURE OSCILLATION.

Orbits. - In simple oscillatory waves each water particle moves in a circular and closed orbit. The water body itself, therefore, has no onward motion. These orbits diminish rapidly with depth, but so long as agitation does not reach the bottom, the orbits are circles at all depths. ${ }^{1}$ The particles on the crest are moving in the direction in which the wave is traveling and particles in the trough are moving with the same velocity in the opposite direction.

Differential movement.- On a line in the direction of the wave movement (hence crossing the waves at right angles) each particle is subject to a gliding between its neighbors. The amount of this gliding is of molecular dimensions, hence not infinitely small. It will be spoken of here as the differential movement of particles. For diagrammatic purposes it is convenient to consider this differential as a considerable arc of the orbit, hence particles are chosen which are removed from one another by a considerable fraction of the length of the diameter.

General form of wave.-In a series of particles moving in equal orbits each particle is more advanced in its orbit (has a more advanced phase) than the one in front of it. If a series

I This principle was clearly elucidated by Gerstner in 1804. This and other fundamental principles of oscillatory wave motion are clearly set forth in the report of the brothers Weber on their experiments conducted in the early part of the last century. See "Wellenlehre, ERnst Heinrich und Wilhelm Weber, Leipzic, 1825. This report also summarizes Gerstner's work and all other previous studies on waves from the time of Newton to 1820 . 
of orbits be drawn and the positions of the several particles be connected by a curved line, that line will show the wave form (Figs. I to 6). The curve is a trochoid. It may be produced by rolling a circle on the under side of a straight horizontal line.
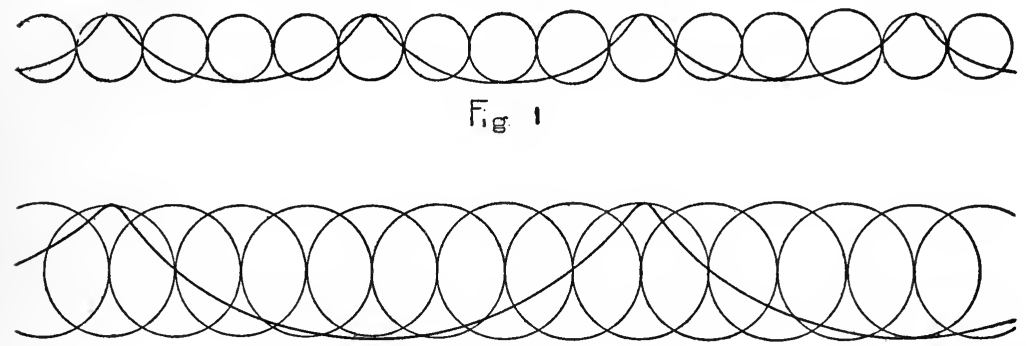

Fig 2.

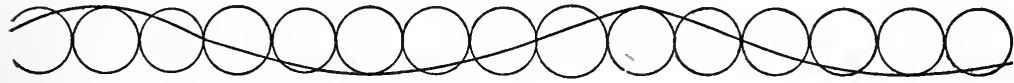

Fig 3 .

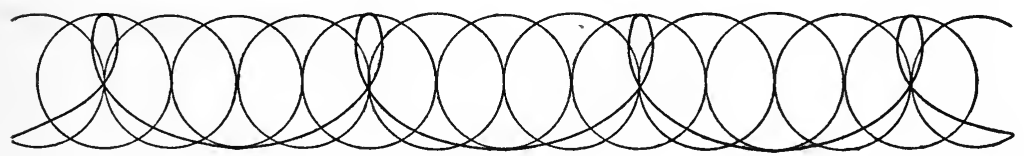

Fig: 4.

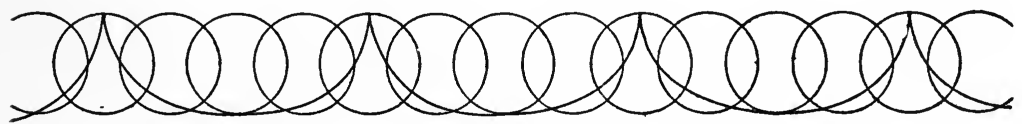

Fig 5

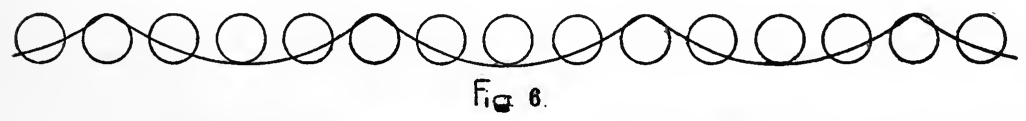

${ }^{x}$ First recognized by Gerstner, Theorie der Wellen, Prague, 1804. See p. 343 of reprint in WeBER's Wellenlehre. Discussed mathematically by W. J. M. RANKINE, "On the Exact Form and Motion of Waves at and near the Surface of Deep Water," Philosophical Transactions, 1863, page I27. 
The path generated by any point within the circle is a trochoid. This line will be sharply curved or broadly curved, approaching straightness, according as the point which generates it is chosen near the circumference or near the center. The distance from the center to the generating point is called the tracing arm. When the point is at the circumference-that is, when the tracing arm equals the radius of the rolling circle-the curve is cusped at the top and is the common cycloid (see Fig. 8). This is the steepest and shortest form which a true wave can have. If the tracing arm be longer that the radius of the moving circle the curve is looped instead of cusped (Fig. 4). The failure of the water surface to assume these looped forms results in breakes.

Steepness dependent on differential movement.-If the same series of particles in their orbits be represented in several diagrams, assuming for each diagram a different amount of differential movement, the wave will be found to be long when the differential movement is small, and short when the differential movement is large (compare Figs. I and 2). If the size of the orbits be increased while the distance between the particles remains the same, and at the same time the differential movement continues to be a certain arc of the orbit, the wave-length remains the same, but its height and steepness increase (compare Figs. I and 3 ). If the size of thc orbit be increased and the differential movement remain the same in absolute amount, instead of the same in arc, the shape of the wave will be preserved and its dimensions increased with the dimensions of the orbit (compare Figs. 2 and 3 ). If the differential movement exceeds a certain limit the curve will loop (see Fig. 4). This condition corresponds to that of breaking waves as noted above.

Movement of particles below the surface. - If a series of equidistant particles be considered which lie in a vertical line in still water, the movement of the topmost or surface particle is represented by any one of the orbits considered above. That of the second one is similar in every way except in size of orbit and hence in velocity. Its orbit is smaller and described in the same 
time. The two particles have always the same phase and hence their movements are parallel at a given instant. The same is true of the third particle and all below it, the orbits decreasing in a descending geometrical progression (Fig. 7). ${ }^{x}$

This fact is to be taken with one above stated, namely, that if orbits be decreased while the angular differential movement remains constant, the sharpness of the trochoid curve is reduced. It results from these properties that in a breaker where the curve of the surface would intersect itself, and is therefore impossible, the trochoids below the surface would show less of looping until a level is reached where normal wave motion is going on (compare Figs. 4, 5 and 6).

Lines of like phase.-If the orbits of a vertical series of particles be represented in diagram (see Fig. 7) and the corresponding points on the circles be connected with lines, then the line connecting the highest points and that connecting the lowest points of the several orbits are seen to be straight and vertical. The remaining lines are curved and inclined. In Fig. 8 these lines of like phase are shown in the positions where they occur in the wave. The particles ranged along any one of these lines would be in a vertical line if the water were at rest, just as all particles on one of the trochoid curves would lie in a horizontal line. ${ }^{2}$

Consequences of the trochoidal form and of decreasing orbits below.-If a horizontal plane be passed midway between the level of the crests and that of the troughs it will pass through the centers of the orbits described by the surface particles. All the water at the surface above this plane will then have a forward component, and all the water at the surface below this plane will have a backward component. An inspection of the diagrams will show that the crests are steeper and shorter than

"RANkine, loc. cit. p. I31. RuSSEll, "Report on Waves made to the meeting of the British Association 1842-3," reprinted in The Wave of Translation, London, 1885, gives formulæ adapted from Gerstner for the rate of orbitical diminution with depth.

2RANKINE, loc. cit., p. I29. 

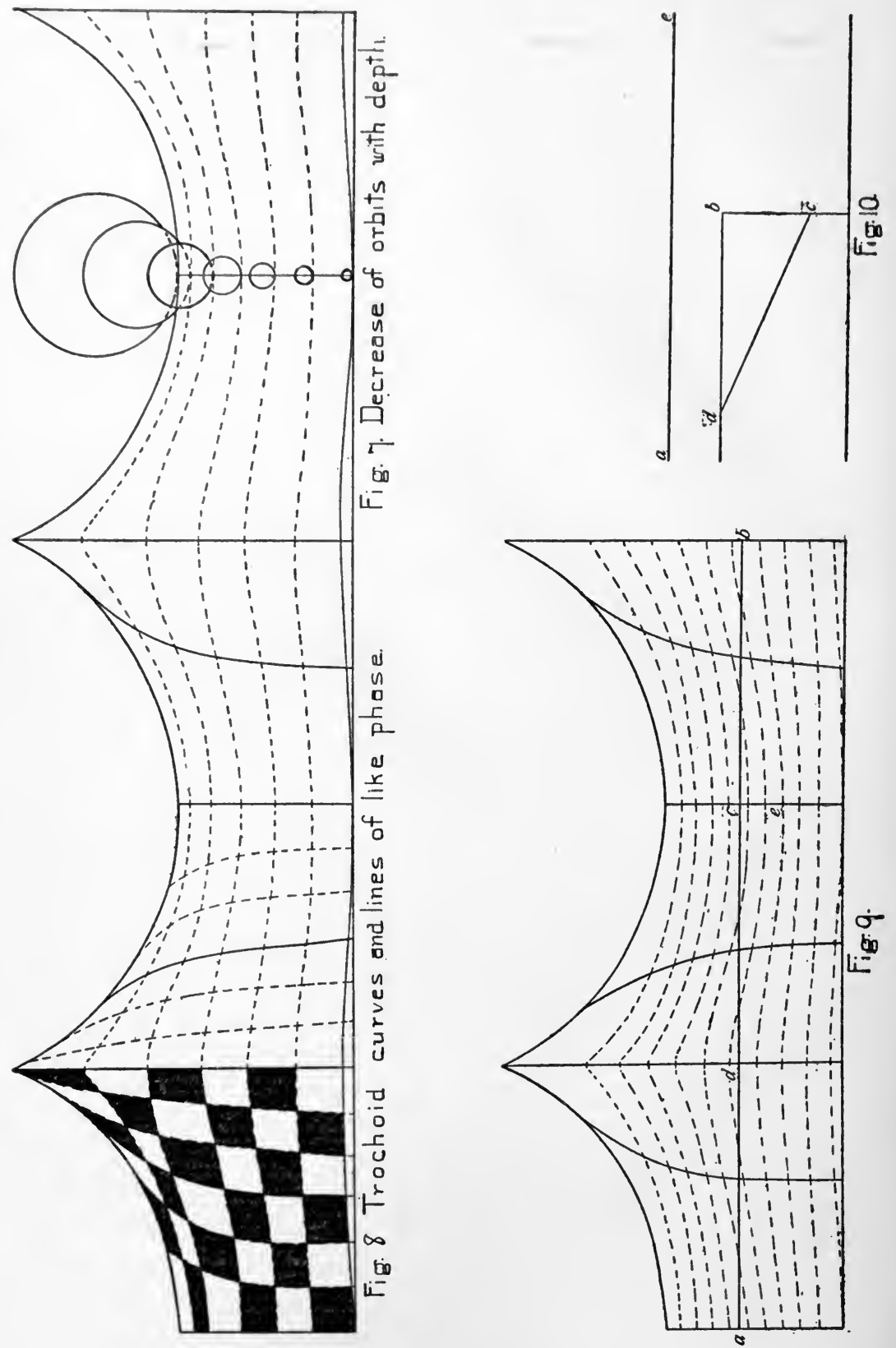
the troughs. This contrast increases as the wave shortens (compare Figs. I, 2, and 5). The crests have not a sufficient volume of water to fill the troughs, and hence the level of the water at rest is lower than the level of the centers of the orbits which the surface particles describe. The lifting of the mean position of particles above their normal level gives a store of potential energy in the wave in addition to the kinetic energy of the motion of the water. It may be shown that this energy of position is exactly equal in amount to the energy of the water's motion. This lifting and this store of potential energy are at a maximum when the wave has its greatest steepness (when it has the cycloid form).

The surface layer is thus divided into strips, in one-half of which the water is moving forward, while in the other half it is moving backward at the same rate. The peculiarity of the case lies in the fact that the backward moving strips are broader than those moving forward. Fig. 8 shows that the same is true in less degree of layers below the surface. Nevertheless, the amounts of water moving in the two directions are equal because of the greater thickness of the layers in the forward moving strips. The contrast of thickness and also of breadth disappears with depth.

\section{MOVEMENTS DURING WIND.}

Effect on size and form.-The immediate effect of wind in the direction of wave movement is to accelerate the movement of the particles on the crest. It also retards the backward motion of those in the trough, but this effect is smaller because these particles are largely protected from the wind. The result is (I) an increase in the size of the orbits; (2) an increase in the differential movement of the particles at the surface; (3) more rapid traveling of crest than trough, hence greater steepness in front. The first would result in increasing both height and length of wave in the same proportion. The second results in greater steepness, that is, a shortening in proportion to their height. The increased differential movement is accompanied by

'Ibid., p. 132. 
increased friction which comes at length to consume all the energy derived from the wind which cannot then further increase the height of the waves. The opposite effects are seen when the wind has ceased. Friction gradually diminishes the differential movement of particles and the size of the orbits. Waves then become lower and at the same time longer in proportion to their height.

Periodical large waves. - The change of wave-length must be propagated downward gradually. If such propagation were immediate, the wave-length at the surface would always be equal to that below. Not being immediate, there may be at times considerable differences in length. The periodical large waves always seen in a storm, may result from composition of lower and upper waves having different periods, as well as by composition of surface waves of different systems.

\section{WAVES IN WATER OF FINITE DEPTH.}

Wave base.-The extent of orbital movement decreases in geometrical progression with depth. A point is therefore reached where the force is too small to overcome the viscosity of the water. Before this point is reached and at comparatively small depths the movement is so slight that it cannot affect the smallest solid particles resting on the bottom. This level, below which the largest waves are inoperative, has been called wavebase. Its depth for any given lake or part of the ocean is a function of the height and length of the largest waves.

Behavior of water above wave-base in pure oscillation.-Before considering the action of water on a bottom which lies above wave-base it will be convenient to examine its behavior at any horizontal plane passed through a system of waves. Referring to Fig. 9, let $A B$ be such an ideal plane. Being above wavebase it is in the region where the "planes of continuity" (planes including always the same particles which are in a horizontal plane when at rest) are in trochoid curves. The lines of like phase are inclined toward the crests; hence the layer of water included between two planes of continuity is not only thinner 
but broader under the troughs than under the crests. In any one such layer the water is moving forward under the crests (point $D$, Fig. 9) at the same velocity with which it moves backward under the troughs (point $E$ of same layer). Of two adjacent layers the lower one is composed of slower-moving water. The line $A B$, drawn in a horizontal plane, traverses higher layers of water under troughs (at point $C$ ) and lower layers under crests (point $D$ ). Therefore the backward moving water along this plane has a more rapid motion than that moving forward. The area covered by it on the horizontal plane is also more than that covered by the forward moving water. This excess of backward movement below is the necessary correlative of the excess of forward movement above, for above the plane traversing the centers of the topmost orbits the movement on all planes is forward.

The same when waves are wind-driven.-If now the water be conceived to be driven by a wind, the current movement produced at any given depth must be added to the forward movement in the corresponding strips which lie below the crests, and subtracted from the backward movement in those under the troughs. The forward and backward velocities in any one layer between two trochoidal planes are now no longer equal. When a certain rate of current is reached, the forward movement in the lower layer traversed by the horizontal plane under the crests (point $D$, Fig. 9) will equal the backward movement in the upper layer which the plane traverses under the troughs (point $C$ ). A certain force of wind will therefore cause a balance of to-and-fro movements at a horizontal plane below the surface. Any greater force will cause an excess of forward motion.

Film representing surface of continuity.-If in one of the surfaces of continuity in a system of waves of pure oscillation, a film could be introduced which is perfectly flexible and frictionless, this film would show alternate depressions and elevations corresponding to those on the surface of the water, but less sharply curved. The curves would progress after the manner of 
surface waves. Any one point in the film would rise and fall vertically; any particle of water adjacent to it would continue to describe its normal circle, gliding to-and-fro on the frictionless film and tracing a straight line upon its surface. The diameter of this orbit is represented by the vertical distance through which any point in the film swings. If the water above the film be viewed in cross section, the area in which it is moving forward would equal that in which it moves backward.

Action on a solid horizontal plane surface.-If the film be supposed now to be stretched to a horizontal plane and to become a solid bottom of the ordinary kind, several changes become necessary in the behavior of the adjacent water particles. The up-and-down movement in their orbits becomes impossible, but the to-and-fro movement, tracing straight lines on the surface, can be continued. Observation shows that this does occur, that particles near a shallow bottom move back and forward in straight lines, and that vertical movement gradually appears in the paths of higher particles, these paths being at first very flat ovals, but becoming higher and more nearly circular as the surface is approached. ${ }^{2}$

The energy of the vertical movement thus interfered with is partly expended in friction on the bottom, though it is quite possible that a part of it may be used in an increased horizontal amplitude. $^{2}$ It is a matter of observation that this flattening of orbits affects the movements of surface particles as well as of those below. ${ }^{3}$ This effect on the topmost orbits is in proportion to the degree of interference at the bottom. Very much elongated orbits indicate large friction, just as circular orbits indicate that there is no appreciable interference at the bottiom.

Effect on wave-length, etc.- The immediate effect of retardation of particles in contact with the bottom must be an increased

'WeBER, Wellenlehre, p. 124.

${ }^{2}$ The observations of the brothers Weber, as recorded in the table given in Wellenlehre, p. 124, seem to show that the horizontal motion on a shallow bottom, while less than at the surface, is actually greater than a certain intermediate point.

3 Weber, loc. cit. 
differential movement of adjacent water particles. The laws of fluids require that this differential movement be distributed throughout the series of particles reaching to the surface, though experienced to a less degree as the distance from the bottom increases. It has been shown that increased differential movement implies decreased wave-length. This shortening, accompanied by steepening, may or may not be sufficient to cause breaking. Since these effects are greater at the bottom than at the surface, the lines of like phase will incline forward. These effects - the increased differential movement, the shortening waves, and the forward inclination of lines of like phase-follow from friction on the bottom, but it is immaterial whether this friction be that of the forward-moving or that of the backward-moving water. The forward inclination of lines of like phase indicates nothing as to the movement of the water as a body. The inclination of these lines may be arrived at in another way. The retarded particles below may be thought of as having a decreased angular velocity, and hence a less advanced phase than the upper particles in the same vertical line. This would require that lines of like phase should connect them with upper particles in advance of them in the direction of wave movement.

Comparison of friction in forward and backward movement.Looked at in cross-section, the area of the backward-moving water above the line $A B$ (Fig.9) is less than the area of forward-moving water. The areas are equal when bounded below by one of the trochoid curves. The area of backward-moving water is made smaller by the substitution of a rigid plane for the depressed part of the trochoid, and that of the forward-moving water is made larger by the substitution of a flat bottom for the curve bulging upward. This constriction and consequent greater friction of the backward-moving water makes itself felt in the form of the wave and in the bodily movement of the water.

Asymmetrical form. - The velocity of propagation of wave crests depends purely upon the behavior of particles in the upper halves of their orbits, while the propagation of troughs 
takes account of the lower halves only. It results from a greater orbital velocity in the upper halves that crests are propagated more rapidly than troughs. ${ }^{x}$ The necessary accompaniment of this is the asymmetrical form, steeper in front than behind.

Resulting currents.-The constriction of backward-moving water mentioned above may be compensated either by greater velocity or by broadening the area of backward flow. Upon either of these assumptions, or upon the assumption of no compensation, certain conclusions follow from a geometrical inspection of the diagram, and these conclusions agree with observed phenomena.

Assume first that the deficiency in backward movement is uncompensated. This assumption involves an excess of forward movement which would be observed as a current, a well-known phenomenon where waves enter shallow water. On this same supposition of no compensation the area of the bottom covered by the backward-moving water is greater than that covered by the forward-moving water, and the velocity of that moving backward on the bottom is greater than of that moving forward at the same depth, because the former, being under the trough, is nearer to the surface. A current of this type would therefore be distinctly a surface feature which would not wash the bottom in the direction of its flow. It would, in fact, involve a certain amount of counter-current at the bottom, independent of any of the conditions which give rise to undertow.

Assume next that the deficiency of area of backward moving water is compensated in one of the ways above mentioned, either by greater velocity or by broadening the area. In either of these cases the backward movement on the bottom will be in excess, and will suffer more interference by friction than the forward movement will. This greater interference with the backward movement will favor, with each oscillation, a residual advance of the water as a whole, causing a progression in the

"Compare also C. S. Lyman, "A New Form of Wave Apparatus," Journal of the Franklin Institute, Vol. LXXXVI, p. 187. 
direction of wave movement, by a process which has something in common with walking. In this way also, pure oscillation would give rise to a current.

It is evident then, that when a system of waves of pure oscillation advances over a shallow bottom, any supposition that may be made regarding the adjustment of internal movements will result in a forward flow of water above, and a dominance of movement in the opposite direction below. Owing to friction, the latter alone is never equal to the former. The resulting movement of water in the direction of wave propagation, whether it be viewed as a current or as an increase of the positive over the negative parts of ordinary waves, is not the same as waves of translation, technically so called. ${ }^{x}$ These latter obey different laws and move with different velocities. They may be occasioned by breakers, or may perhaps grow out of oscillatory waves by gradual transition, but their movements are characterized by certain features to be mentioned later.

The return current.-As soon as a current is initiated a return of the water becomes necessary. If the process described above be supposed to take place on a shoal without shores this return may take place by another route. In this case the current may proceed as described for an indefinite time. If there is no return over another area by horizontal circulation, then the return must be over the same area by vertical circulation; that is, either above or below the original current. If the forward orbital movement above exceed the backward orbital movement below, as seems necessary, and no lateral escape is at hand, the pressure due to increased height of the water would cause a counter current which would appear below as undertow.

Action on bottom materials. - The essential value of the consideration of these currents, springing from waves of pure oscillation, is in the necessary conclusion that the work of such waves is backward at the bottom, and not forward. The advance of the water described is due to interference with its backward flow. The same friction which impedes the backward movement of the

\footnotetext{
'J. Scotr Russell, The Wave of Translation.
} 
water causes the motion which the water loses to be communicated to the materials of the bottom. The case is roughly analogous to the wheels of a locomotive, which in "flying the track" brush the sand on the track backward.

The case of wind-driven waves. - The above case is applicable only to waves of pure oscillation, which have of necessity been generated in deep water and are advancing over a shallow bottom. If the wind is blowing at the same time in the direction of wave movement, the result will be similar to that found in considering a mathematical plane above wave-base, provided, of course, that the return of the water is by horizontal circulation. The action of the wind increases the forward motion under crests and diminishes the backward motion under troughs. When the effect of this action reaches a certain amount, the influences named above, which give dominance to the backward movement at the bottom, will be counterbalanced, and any greater effect of the wind will give, at the bottom, an excess of forward movement. A moderate effect of the wind is probably usually sufficient to overcome the backward brushing due to oscillation alone. If the return is by vertical circulation, any increase in current above involves an increased reverse current below.

The case of breaking waves.-When waves generated in deep water advance over a bottom sufficiently shallow to cause breaking, a new factor is introduced. In this case there is a tendency to the formation of positive waves of translation, which may sometimes develop typically, though doubtless more often their motion enters in merely as a component. It is in the nature of these that all the particles in and under the wave form move forward and not backward, and the forward motion is the same at all depths. ${ }^{x}$ To the extent that this factor enters, the effect on the bottom will of course be to urge material in the direction of wave movement.

'See Russell, The Wave of Translation, p. 42; Report on Waves, p. 307 ; also D'AURIA, "A New Theory of the Propagation of Waves in Liquids," Journal of the Franklin Institute, 1890, p. 460 . The last named is a mathematical discussion. 


\section{WAVES IN SHALLOWING WATER.}

Tendency to enlargement of orbits.-When a system of waves generated in deep water reaches shallow water, certain forces operate to increase the sizes of the orbits, while others produce the opposite tendency. In general the increase of orbital motion is due to the transmittal of the motion of a larger amount of water to a smaller amount. ${ }^{x}$

If the shallow water be separated from the deep water by a vertical face ( $B C$ in Fig. 10), the change may operate in some manner similar to the following: The deep water on the right side of the figure is agitated to the depth of $C$ by waves traveling toward the left. The motion of particles below the level of $B$ is influenced by the vertical face $B C$, this influence being greater in proportion to their nearness. Those in contact with the surface must move in straight lines up and down, while those farther away describe ovals whose longer diameters are vertical, and whose shapes become more circular with distance from $B C$. The energy of the horizontal motion thus lost is, of necessity, partly expended in friction on the vertical face. That which remains must be devoted to increasing the vertical movement. By this means it is again communicated to the particles above the level of $B$.

If the change from deep to shallow water be gradual, the analysis of the process is essentially the same. In this case, however, the circular orbits below will give way to straight line movement, not vertical, but parallel to the sloping bottom $D C$. As before, friction will consume a part of the energy which orbital motion has lost, the remainder being expended in increased movement parallel to the sloping bottom. Of this movement the vertical component will go to increasing the vertical axis of the orbits above.

Tendency to diminishing orbits.-Along with the above tendency to increased orbits come two tendencies toward diminution. The first of these is the influence of the flatter orbits of the lower particles. It tends to diminish the vertical movement

' Compare C. S. Lyman, loc. cit., p. I93. 
above, but not the horizontal. The second influence toward diminution is the friction on the bottom which is shared by the particles above.

Opposite tendencies simultaneous.- On a sloping surface the opposing tendencies act at the same time. It is evident that in proportion as the slope is steep, sudden enlargement will be favored, and that slow shallowing favors reduction in size because of the long continued action of friction. Theoretically, there should be a grade on which an incoming wave should suffer no change of height, but since the form and internal movements would change, this ideal grade is not of importance in considering the work of water on the bottom.

Tendency to decreased wave-length. - If the supposed tendency toward orbital increase be balanced by the opposite tendency arising from friction, there will, of course, be no increase in the length or height. However, when waves do increase in height, showing that the orbits have enlarged, they are still very commonly diminished in length and of necessity increased in steepness. This is readily explained by the increased differential movement of particles, initiated by friction on the bottom.

Tendency to steepening due to wind.-The largest on-shore waves usually act in conjunction with the wind blowing in the approximate direction of their movement. The effect of wind on waves in deep water was seen to be similar to the effect of a shallow bottom, namely, (I) increase of orbits ; (2) increase of steepness; (3) asymmetrical form. These effects may be carried to the point of breaking, even in water of infinite depth (whitecaps). On a shallow bottom the effects are increased by the concurrent action of the two factors. Where there is no wind waves are commonly supposed to break in water whose depth is equal to or a little greater than the height of the waves above the level of repose. ${ }^{x}$ When waves advancing on a shallow bottom are already strained by the wind, they may break with much regularity in much greater depths of water, equal to perhaps two, three, or four times the height of the wave. Thus while the

$\therefore$ Russell, Report on Waves, p. 245. 
breaker line for waves without wind is far up the slope from wave-base, it may move down indefinitely near to wave-base when the wind is active.

Tendency of wave to recover form.- Suppose a system of oscillatory waves to advance toward a shelving shore until the wave-base intersects the bottom. One effect must be produced here regardless of qualifying conditions. Bottom friction begins and that involves increased differential movement of particles, which is accompanied by shortening and steepening of waves. This implies increased internal friction, which in turn, operates to decrease the orbital motion and therefore wave dimensions. In so doing it would take away the conditions of bottom friction and its results. The wave would then return to its deep water form. Thus there is a chain of consequences from the original interference at the bottom, which involves at first the change of wave form, but later a restoration, the final result being reduction in dimensions only, suited to the diminished depth. Another decrease of depth must then be assumed if the wave be supposed to continue its contact with the bottom. Thus there is a certain minimum slope for the bottom, upon which the waves may be propagated as a shallow-water wave. In so far as the wave is affected by increase of orbit due to diminishing amount of water, the effect will be to hasten the deformation and to retard the recovery of form. If the wind is active it would retard the decrease of orbital movement and the minimum slope mentioned would be smaller.

Limit of tendency to recover form.- The greater the reduction of depth, the greater the increment of internal friction tending to reduce the wave size, and the greater this friction, the more rapidly does it operate to accommodate the wave dimensions to diminished depth. This corrective tendency has, however, a limit. This limit is marked by the breaking of the wave. There is, therefore, a certain maximum slope for the bottom upon which the wave may be propagated without breaking; at or beyond this maximum the wave breaks and other agencies come in. The effect of wind as before, is to diminish the maximum slope; hence 
true breakers (not whitecaps merely) may occur during a wind on a shore where waves of the same size would not break in a calm.

Effect of breaking on wave propagation.-Even when the distortion of wave form has been pressed beyond the breaking point, the effort to recover its form and habit does not cease. This effort is now favored by all the tendencies which existed before breaking and re-enforced by one more arising from the falling crests. As shown in the diagram (Fig. 4), breaking is an expression of conflicting orbits. The water above the node of the hypothetical surface does not continue the curve which it has been describing, but falls confusedly on the front of the wave. Here its downward motion is in direct opposition to the upward motion of the water in front of the crest. Thus, to the molecular resistance of friction, is added mass conflict, both of which operate to reduce wave motion. This reduction is therefore accomplished more rapidly than in the case of unbroken waves. It results from this, that waves often break at some distance from shore, and after traveling a short distance with foaming crests, recover their form and advance a long distance with crests entire. There is a certain slope on which waves will advance with nearly uniform shape and continuously foaming crests. On a gentler slope they will recover their unbroken form; on a steeper slope the first breaking occurs close to shore, and the wave form is speedily lost.

Waves of translation.-When waves of oscillation enter shallow water the habit of the water particles changes and becomes a compromise between orbital oscillation and movement of an entirely different nature, belonging to waves of translation. ${ }^{x}$

The essential features of the positive wave of translation, known also as the wave of the first order or the solitary wave are, (I) it is initiated by an elevation of the water surface above its normal level; (2) it is propagated without a corresponding trough and without companion crests, being entirely above the undisturbed level of repose; (3) its rate of travel is greater

'RUSSELl, Report on Waves and Wave of Translation. 
than that of waves of oscillation, when like wave-lengths are assumed, the two rates having about the ratio of three to two $;^{x}$ (4) the water particles move forward and not backward, starting from rest as the wave approaches and coming to rest when the wave has passed; (5) the forward motion of particles at all depths is the same and equal to the volume of the wave divided by the depth of the water; (6) the paths of the particles are semi-ellipses in a vertical plane, the major axis being the distance through which the particle moves forward, and the minor axis varying from zero at the bottom to the height of the wave at the surface. This movement is in no sense the same as that of wind-driven waves or any other oscillatory wave motion compounded with current. It usually coexists with the latter on shallow bottoms, resulting in waves of a hybrid kind; but waves of nearly typical translatory character may sometimes be seen in nature. Whether the waves be of a pure or mixed type, the essential fact here is that a new factor has entered, whose action at the bottom is different from that of oscillatory waves and from that of currents.

The fact of this change to translatory character on a gently sloping beach may be seen in the behavior of floating chips. which are seen to move forward on crests but not backward between crests. In place of the trough proper is a wide strip. whose surface is almost flat and the water of which is standing still. The laws of translatory waves require that they move more rapidly than the oscillacory. This might be expected to reveal itself in broadening intervals between crests as waves take on the translatory character. It is probable that this may occur under suitable conditions. The tendency is usually more than counterbalanced by two factors. The first is the decreasing depth. which is the main factor in controlling the velocity of waves of translation. The second is the increasing strength of undertow near shore which retards the translatory movement at the bottom.

As to the manner in which this new habit is developed, it may ' Tbid., p. 288. 
be cited that perfect waves of the first order are produced experimentally by the sudden addition of water at one end of a rectangular vesscl, or by the immersion of a solid, or by a sudden pushing forward of the wall of the vessel, the effect in each case being the local raising of the water surface above the level of repose. A corresponding process in lakes or sea where the bottom becomes shallow may be found in the sudden delivery of the mass of water which falls upon the front of a breaking wave. Observation on the shores of large water bodies, such as the great lakes, would indicate that the area over which waves show a translatory element is somewhat definitely limited by the breaker line. It is probable, however, that there is also a more gradual change by which the waves become increasingly positive as the water shallows and the features of waves of the first order are thereby assumed.

If the modifications of oscillatory waves in shallowing water be reviewed while holding in mind the charactertistics of translatory waves as given above, it will be observed that these changes are all in the direction which would favor the conversion of oscillatory into translatory waves. This is seen in the increase of crests with corresponding disappearance of troughs; the growing excess of the forward movement of particles over backward movement and the increased horizontal amplitude of the lower orbits, approaching equality with that of the orbits above. For present purposes it may suffice to adopt the conception of $\mathrm{Mr}$. Russell ${ }^{x}$ who thought of the overgrown crest as

s The wave of the second order may disappear and a wave of the first order take its place. The conditions under which $I$ have observed this phenomenon are as follows : one of the common sea waves, being of the second order, approaches the shore, consisting as usual of a negative or hollow part and of a positive part elevated above the level; and as formerly noted, this positive portion gradually increases in height and at length the wave breaks, and the positive part of the wave falls forward into the negative part, filling up the hollow. Now we readily enough conceive that if the positive and negative parts of the wave were precisely equal in height, volume, and velocity, they would by uniting, exactly neutralize each other's motion, and the volume of the one, falling into the hollow of the other, give rise to smooth water; but in approaching the shore the positive part increases in height and the result of this is to leave the positive portion of the wave much in excess above the negative. After a wave has first been made to break on the shore it does not cease to travel, but if the 
falling forward into the diminished trough in the act of breaking; the trough is more than filled and the excess of water initiates a wave of translation exactly as in Mr. Russell's experiments.

Volume of undertow. - It is not necessary to suppose that the loss of velocity of the undertow is as rapid as the increase of its cross-section. This would be the case if all the upper water moving shoreward should reach the shore before turning back. The volume of the undertow would then also be constant throughout its course and its velocity would be inversely as its cross-section. But even if the loss of motion due to friction and interference of the bottom be ignored, not all the shoreward moving water reaches the shore. The on-shore motion causes. elevation of level over a belt of considerable width. This broad elevation constitutes a head which is the cause of outward flow below. It may be shown that the average position at which incoming particles turn back and join the undertow, is at the center of mass of the head. This head is greatest at the edge of the water, hence more water turns back at that point than at any other, but the undertow which has its beginning here is constantly being augmented by that which returns toward deeper water without reaching the shore.

slope be gentle, the beach shallow and very extended (as it sometimes is for a mile inward from the breaking point, if the wave be large) the whole inner portion of the beach is covered with positive waves of the first order, from among which all waves of the second order have disappeared. This accounts for the phenomenon of breakers transporting shingle and wreck and other substances shoreward after a certain point; at a great distance from shore or where the shores are steep and abrupt the wave is of the second order, and a body floating near the surface is alternately carried forward and backward by the waves, neither is the water affected to a great depth; whereas, near the shore the whole action of the wave is inwards, and the force extends to the bottom of the water and stirs the shingle shoreward; hence the abruptness also of the shingle and sand near the margin of the shore where the breakers generally run. . . . The residuary waves given off after breaking are wide asunder from each other, are wholly positive, and the spaces between them, several times greater than the amplitude of the waves, are perfectly flat and in this condition they extend over wide areas and travel to great distances. These residuary positive waves evidently prove the existence, and represent the amount, of the excess of the positive above the negative forces in the wind wave of the second order.-Report on Waves, p. 292. 
Relation of the phenomena above to agitation on the bottom.It is to be inferred from what precedes that symmetrical wave form indicates freedom from interference at the bottom, that friction below is great in proportion as crowding, steepening, and asymmetrical form above are prominent, and that where an off-shore breaker line is seen it indicates a maximum of bottom interference. It is understood in all cases that the surface effect will lag a little behind the cause below, and therefore appear a little to shoreward.

\section{PROFILES RESULTING FROM FORCES DISCUSSED ABOVE.}

In the actual operation of the forces discussed above, the resulting action on a sloping bottom may be outward at all places, or inward at all places, or outward over one part and inward over another. Forces in either direction may be graduually augmented or diminished. The different forces are capable of different combinations. Each set of conditions will lead to certain features of profile. If there be no change of condition, a permanent profile of equilibrium may be reached. The constant supply of load constitutes an ever shifting condition. Equilibrium as commonly realized depends on the uniformity of this supply.

Factors in profile-making.-The agencies which shape the marginal bottom may be treated in three groups, (I) oscillatory wave action and undertow, carrying material from shore; (2) on-shore currents and translatory wave action, carrying the material toward the shore; (3) currents alongshore. The tendency of the first group is to steepen the slope from the water's edge to the line at which its erosive power ceases, and deposition begins and to reduce the slope beyond that line. There is also for the second group a line of maximum power on the bottom, within which their effect is to steepen the profile by accumulation at the water's edge, and beyond which the slope is reduced by cutting down. Currents alongshore will be introduced later.

Conflict between on-shore and off-shore action.-The first two 
pairs of agencies are in conflict as to the direction in which bottom materials are to be moved. If all the water which moves shoreward must return over the same area and as a bottom current, this current would seem to have greater efficiency than the one above, moving in the opposite direction. This is certainly the case where translatory waves are not favored, as where the off-shore slope is steep. Where slope is gentle and translatory waves are well developed, they have one decided advantage. They are short as compared with the distance from wave to wave, hence all the shoreward movement of the water is concentrated into a small portion of the entire time. Divers are said to feel the passing of one of these waves as a sudden jerk between intervals of quiet. The undertow, on the other hand, has a steady flow except as interrupted by these sudden reverses. ${ }^{x}$ The laws of energy give to these concentrated movements a much greater efficiency than to the same amount of motion more evenly distributed in time. On many shores of gentle slope, sand is worked landward, and in this process the agency just mentioned is doubtless important. The effect here referred to is that of waves of translation and is therefore inside the breaker line. It might accumulate sand on-shore but not in off-shore barriers. The dominance of shoreward action is essentially temporary (omitting currents alongshore from consideration). Its effect is to steepen by narrowing the slope. This steepening, in turn, is adverse to waves of translation.

Laws of equilibrium; eroding currents.-Ignoring the presence of a bank and the load derived from it, a current of uniform power tends to reduce the bottom to a level surface, that is, to require equal depth throughout. Equilibrium cannot exist on a level bottom where the power of the current is unequal at different places. In such cases, the depth must suffer a corresponding change until the power of water on the bottom is

"HenRy Mitchell, "On the Reclamation of Tide-Lands and its Relation to Navigation," Report of the U.S. Coast and Geodetic Survey, 1869, Appendix 5, p. 85. In this paper Mr. Mitchell takes the extreme view that the sea restores to the continent "all the material washed from its bluffs and headlands." Certain exceptions are made for islands. 
everywhere the same. A current of uniformly increasing power requires a uniformly increasing depth, that is, a plane slope. The opposite is true for a current of uniformly diminishing power. A current whose power is augmented at an increasing rate, as, for example, in geometrical ratio, requires a descent to deep water on a curve which is convex upward. Increase of power at a diminishing rate requires concavity. Loss of power at increasing rates, and loss at diminishing rates, require concavity and convexity respectively.

Uniform cutting or building.-If a uniform current on a level bottom has eroding power, the whole will be cut down at the same time, and the bottom will remain level while depth increases. In this case the load is furnished at all points equally, and is all carried forward at the same rate. If load be furnished in excess of carrying power, and at all points uniformly (as from top or sides), then the level surface of the bottom would be preserved while depth would decrease.

Load derived from the shore. - To make the case applicable to undertow, the excessive load must be supposed to be furnished at the end where the current enters upon the bottom in question. In this case deposition will first reduce the load at the end upon which it enters and at the same time reduce the depth and thus constrict the current, increasing its power. The latter influence will determine a higher level to which the bottom will be built; a level at which the power of the water is sufficient to carry the load which before was excessive. Filling will then advance forward over the bottom, the filled and unfilled portions both being level, the former growing while the latter diminishes, and the two being separated by a slope, mentioned below. It is evident that the depth at which this slope begins is determined jointly by the power of the water, the amount of the load, and the size of the fragments which make up the load.

The front.-The shape of the slope which intervenes between the area which has been filled and the bottom beyond, will be determined by the rate at which the power of the current decreases. If the loss of power were instantaneous, the slope 
would be simply the subaqueous earth slope. If it be in any arithmetical progression, the slope will be a plane whose steepness will vary with the rate of decrease, the slope being steeper when the rate is higher. If the loss of power be in some other manner than by arithmetical difference, the slope will show a curve which will be convex or concave according as the rate of decrease is augumented or diminished. In actual deposition by a current advancing into deep water, the decrease of power is at an increasing rate, as may be seen from the following. If a plane slope be assumed, so that depth increases in arithmetical ratio, then the velocity of the current will decrease in similar ratio, but transporting power varies as the square of the velocity, hence its rate of decrease is progressively augmented. This will require convexity of slope, a feature generally observed at the edge of embankments and subaqueous terraces. The general law of equilibrium, as given above for an eroding current still applies; current power is uniform over all parts of the bottom, if by the term current power is understood power with referrence to load and the current considered is the resultant of all conflicting currents. In this case, while the current is acually losing power, the loss is balanced by the coincident loss of load, and the uniformity of power in comparison with load is maintained.

Presence of a bank; equilibrium on a slope.-The presence of a bank fixes not only a horizontal limit to the bottom in question, but determines that at this limit the depth shall be zero. This involves a slope. If equilibrium is to exist on this slope in harmony with the general law stated above, the advantage in power due to shallower water on one side must be balanced in one of four ways, (I) the equality of transporting power in deep and shallow water may be partially maintained by the participation of more water where the depth is great than where it is small. In the case of undertow this has been shown to be true; (2) currents in both directions may be stronger, so that the resultant motion in one direction may be more in shallow water than in deep water, it may even be zero or it may be in the opposite direction. The factors of translatory wave motion and on-shore 
currents may occasion this condition; (3) the excessive power of the water on the shallow bottom may be employed in the transporting of a greater load or even in erosion. This is quite generally true; (4) the material may be heterogeneous, the larger stones coming to rest in the shallower water because of their ability to withstand the greater agitation at a higher level. Of all these reasons, it will be seen that only the first can provide for a permanent slope: the others depend upon a continual supply of fresh drift.

Necessity of a continuous supply of load.-Suppose now that a short section of coast line be enclosed between perfectly resistant walls or piers perpendicular to the shore line, and extending out to deep water. The transportation of material alongshore will thus be prevented. If the shore also be supposed to be perfectly resistant, so that no new drift can be furnished to the waves, then the profile of equilibrium, toward which the bottom will tend, is a steep descent from the water line to the depth at which undertow becomes ineffective, and then a low slope outward, following the base of effective undertow. This base is necessarily on a slope because of the increasing volume of undertow with distance from shore.

Effect of a supply of drift.-If now, drift be supplied at the shore line at a given rate, filling will occur at the foot of the steep descent leading down from the water line, until the bottom has risen to a level at which the power of the water is sufficient to transport the material at the rate at which it is furnished, and this filling will advance off-shore, ending in a convex front as shown above.

At the shoreward boundary of this filling area is an angle made by the plane of deposition, with the steeper descent leading down from the water's edge to the line at which deposition becomes possible. In an actual case, where the material of the shore yields to erosion, the water's edge is carried landward, and the first descent is not only far from vertical, but in weak material, is very gentle; probably always steeper, however, than the slope made by deposition farther out. This may be observed 
on almost any of the coastal charts of the United States Coast and Geodetic Survey. The east coast of Florida furnishes typical illustrations.

Normal profile; cutting coast.-The normal profile then, of a shore where the resultant of transporting power is outward, is a compound curve, which is concave near the shore, passing through a line of little or no curvature, to a convex front. Where this front rests upon the bottom below the reach of currents, the descent merges into the more level bottom by another concave curve, due to deposition from suspension. If the supply of material from the shore be cut off, the entire shelf will be cut down and its slope reduced and it will necessarily be separated from the shore by a steeper slope than before. If, on the other hand, the supply of material be suddenly increased, a smaller shelf will grow from shore on the surface of the older, for the reason that the new load, being greater, is in equilibrium with the currents at a higher level than before. The greater the load, the nearer will the surface of deposition approach that of the water. On the Atlantic coast of the United States, the depth at which the concave curve merges into the plane of deposition varies from three fathoms near the mouths of some rivers, to ten or twelve fathoms where the lead is smaller. On some parts of the Pacific coast, where the lead is small, the concave curve descends to twenty or thirty fathoms.

Normal profile; building coast.-If the resultant of shore action. be to carry material landward, the general character of the resulting curve cannot be very different, since this process also produces steepening near shore. In general the velocity of shoreward motion increases with nearness to land. If the effectiveness of this motion increases with its velocity, there is no accumulation until the shore is reached. The shore is then progressively steepened by accumulation, until the force which acts shoreward can no longer carry material up against the growing component of gravity. This landward urging of sediments is commonly thought to be one of the factors in the production of off-shore barriers. It is plain, however, that 
unless the power of inward transportation is decreased before reaching the shore, no barrier can form. This decrease may, at times occur, for carrying power will depend not only on the velocity, but on the agitation of waves at the bottom. It has been seen that waves are rapidly reduced in size and vigor in the act of breaking. It is possible, therefore, that when the slope is so gentle that waves recover their form after breaking, thereby showing that oscillatory wave motion has been much reduced, deposition may take place along the line of wave reduction, which is essentially the breaker line. With these conditions alone, however, the growth of this feature would probably be confined to narrow limits by the undertow. It would, moreover, be a very transient feature, a mere incident in the process of shoreward transportation. The steepening of the shore, to which this process is incidental, would rapidly remove the conditions of the incident.

Variations of the compound curve.-The compound curve will be more marked in proportion as the surface of deposition is broad and its slope is gentle. Where it is narrow its significance may not appear from a profile drawn from widely spaced soundings. ${ }^{x}$ If all the waste from the land be carried alongshore, the marginal terrace is of the cut type purely, in which the compound curve is not noticeable, the only prominent angle being that where the surface of cutting intersects the original steeper bottom.

Currents alongshore.-If the effect of currents alongshore were the same at all distances from land, they might be ignored as a factor in profile making. Their variation in strength at different distances from shore produces important results. It has been stated above that for any one current the power at the bottom with respect to the load must remain constant. It may also be shown that of two currents, each of which is furnished with load to its full capacity, the stronger, which may be supposed to dissipate gradually, will be in equilibrium with its load at the smaller depth. Hence if transportation alongshore be

× This is illustrated at many places on the Pacific coast of the United States. 
distinctly greater in a zone adjacent to the land, a smaller terrace will rest upon the larger. If transportation parallel to the shore line be distinctly greater in a zone off-shore, and the supply of drift be at hand, a ridge will be built along the line of this more effective current.

Barriers. - It has been shown above that when the off-shore slope is too low for equilibrium, and there are no currents alongshore, steepening is effected, in the main, by accumulation at the water's edge, though there may be some small tendency to accumulation at or just within the breaker line. When currents are flowing, they have a zone of greater efficiency along this same line or just outside. This is because the material which they transport is more agitated by wave action, and is to some extent lifted into the current. Excessive transportation along this zone initiates the ridge which may continue to grow until it assumes the functions of the beach. It this then called a barrier.

The essential function of the barrier is to steepen the bottom slope by carrying the shore line farther out. If the slope is not abnormally low, the barrier is not needed; nor are the conditions present which make its formation possible, one of these conditions being that the agitation on the bottom at the breaker line should exceed that nearer shore. It was seen above that this condition is present, only on a deficient slope.

The slope may become deficient in several ways. The currents themselves might be the cause; or it may result from the sediments delivered by streams, as at many places on our Atlantic coast; or the gentle slope may have belonged to the original bottom over which the waters rose, as seems to have been the case with Lake Michigan in its former extension in the vicinity of Chicago. Doubtless far the most frequent occasion of deficient slope is the falling of the water level or the rising of the shore. That the immediate off-shore slope should in this case be too low, is the necessary consequence of the concavity of the normal slope near shore. The slope from the Atlantic shore line, where well removed from rivers, as on the 
east coast of Florida, is perhaps ten fathoms in the first two miles, but if the sea level should fall ten fathoms, or the land should rise by that amount, the new ten-fathom line would lie many miles off-shore, and new barriers might be expected. On some of the small lakes of Wisconsin, especially those without outlet, as Silver Lake of the Oconomowoc group, the falling level has found a deficient slope and barriers are constructed.

The front of the marginal shelf.-If the marginal shelf be a pure wave-cut terrace with no addition by deposit, its limit will be marked by an angle where the plane of the shelf meets the original bottom. The depth of the shelf at this edge will constantly approach wave-base, for it may be safely assumed that wherever waves can agitate, there will be sufficient current to transport. If there are currents strong enough to erode below wave-base, the shelf may be cut still lower. The hardness of the rock can make no permanent difference. This is well illustrated even in so young and small a body as Lake Mendota at Madison, Wis., where the sandstone shelves southwest of Governor's Island and Maple Bluff are cut to the same depth as the clay shelves west of Picnic Point and Second Point. ${ }^{x}$

If the shelf is being broadened at the same time by materials carried across and deposited on its front, there will be, between its upper surface and its steep front, a curve convex to the sky as shown above. This steeper slope begins, not at the depth where the power of the water ends, but at the depth at which the power of the water becomes insufficient to carry the entire load. From this depth the slope becomes progressively steeper to the depth at which the movement of the water is ineffective. Off the Atlantic coast of the United States, the depth at which the slope begins to steepen is usually fifty or sixty fathoms, but the maximum of steepness is not attained until a much greater depth is reached. The depth familiarly assigned to wave-base along this coast is one hundred fathoms, and this figure expresses fairly well the horizon at which the maximum steep-

'See hydrographic map issued by the Wisconsin Geological and Natural History Survey. 
ness is reached. This would mean that currents become unable to carry the whole load at fifty or sixty fathoms, and at one hundred fathoms or less, become unable to transport anything except in suspension. If the factor of transportation in suspension did not enter, the front of such a shelf should show the subaqueous earth-slope.

It is commonly assumed as above, that undertow and wave agitation lose their efficiency at the same point, the limit of the former being determined by that of the latter. Probably this is very generally true; moreover, since wave oscillation decreases with depth in geometrical ratio at a high rate, and the decrease of its agitating power is at a rate measured by the square of this same ratio, it may readily be seen that there is a somewhat definite horizon below which wave action is ineffective. Such a condition is signalized by a somewhat definite limit to the sedimentary shelf.

Transportation beyond wave-base.-The undertow may, however, be constricted laterally and preserved from dissipation, as when the water drifts into a re-entrant curve of the shore; or deep currents may result from a system of rebounds. By either of these means the power of the lower water may be increased, so that at depths greater than that of wave-base sand or even gravel may be transported. ${ }^{x}$ In such cases no break in the profile may be seen at wave-base. Broad sheets or streaks of sand may cover the bottom to depths far beyond this line. Such troughs as those of the great lakes, in which all the surface water may be drifted simultaneously in one direction, should especially favor vertical circulation with vigorous movements below. Wave-base of Lake Michigan, where revealed by a sharp angle at the edge of a marginal terrace, is sixty or seventy feet below the surface; yet around much of its margin, a sand covered or gravel covered bottom, concave upward, extends outward to several times this depth with little or no evidence of change of slope at wave-base. ${ }^{2}$ This is to be expected from the

"See H. C. Kinahan, "The Beaufort's Dyke off the coast of the Mull of Galloway," Proceedings of the Royal Irish Academy, Third Series, Vol. VI, No. I.

'Charts of Lake Michigan, War Department. 
necessarily powerful undertow. In Lake Mendota, where wavebase is not lower than twenty feet, sands and even heavy gravels are irregularly distributed over the bottom at depths frequently approaching fifty feet. Some lie at the bases of steep slopes which gravity may have helped them to descend, but others are far from slopes and plainly illustrate the erosive power of currents resulting from a concentration of movement along certain lines.

N. M. Fenneman.

The University of Chicago.

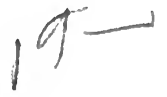




\section{UNIVERSITY OF CALIFORNIA LIBRARY BERKELEY}

Return to desk from which borrowed.

This book is DUE on the last date stamped below.

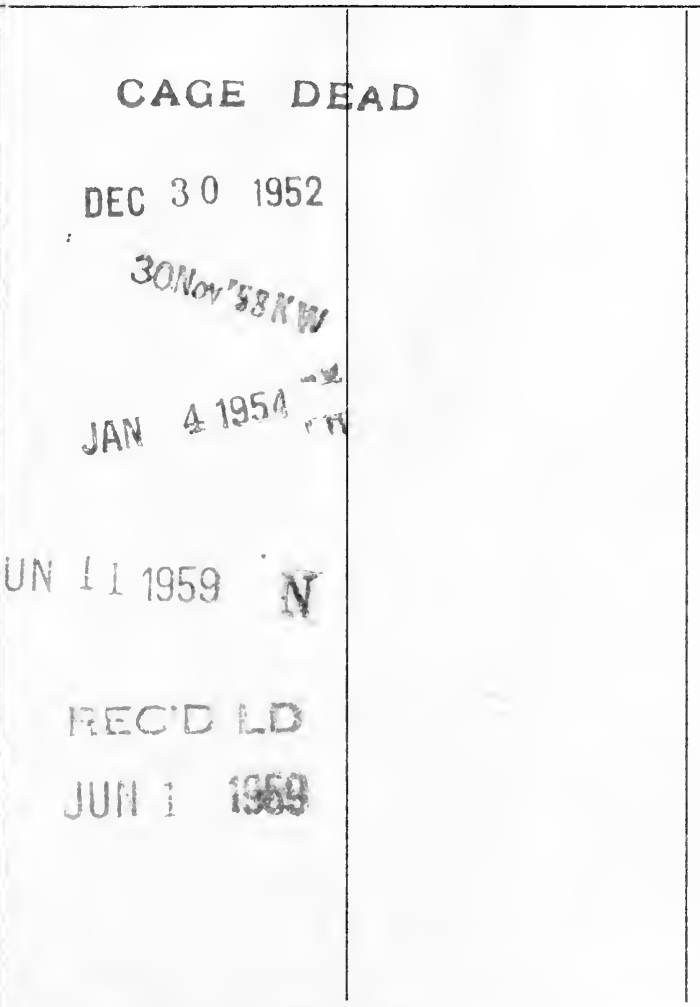




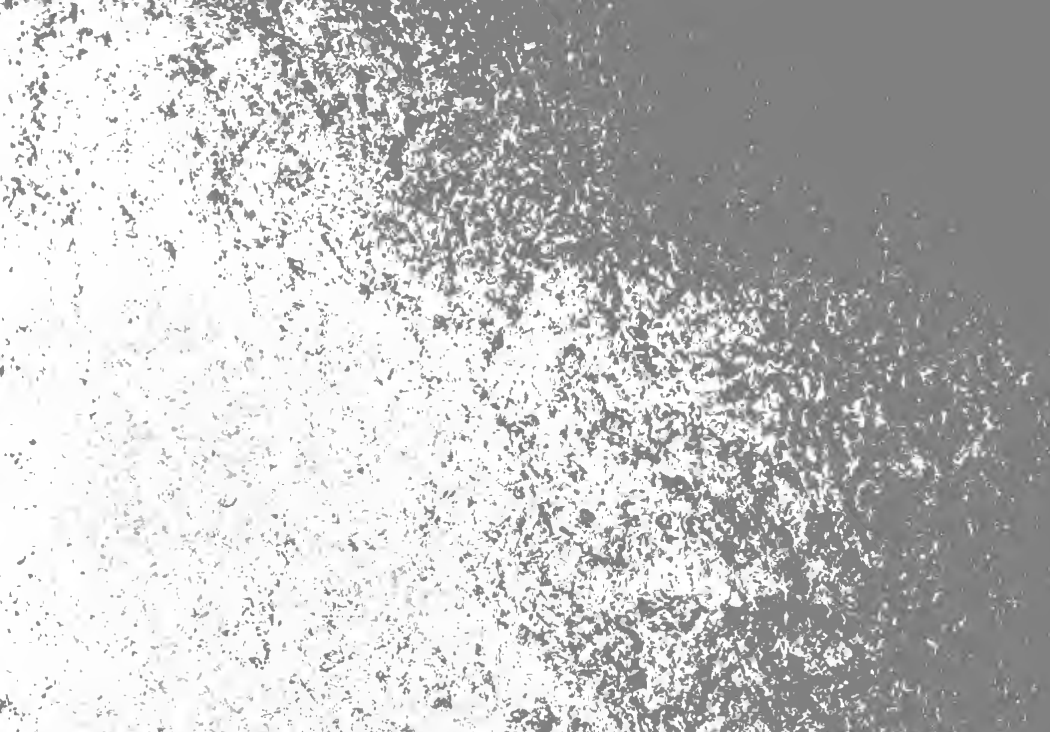

